\title{
IMITATION IN ANIMALS: EVIDENCE, FUNCTION, AND MECHANISMS
}

\author{
THOMAS R. ZENTALL \\ Department of Psychology, University of Kentucky, \\ Lexington, Kentucky, USA
}

\begin{abstract}
The terms social learning and social influence have been used descriptively and theoretically to characterize a broad range of animal behavior from physical antipredatory adaptations such as eye spots, which are totally under genetic control, to the human capacity for the exaggeration of individual characteristics, known as caricature, which are largely under cognitive control. In the present review, the various forms of social influence and social learning are identified and distinghished from imitation, a term that generally has been reserved for behavioral matching that cannot be accounted for using simpler specifically predisposed, motivational, or learning mechanisms. It is suggested that much of the ambiguity in the literature concerning the various forms of social learning can be attributed to the distinction between the function of a behavior and the mechanisms responsible for its occurrence. Finally, the various mechanisms that have been proposed to account for imitative learning are presented and an attempt is made to evaluate them.
\end{abstract}

When the term imitation is used by psychologists it typically implies more than the simple reproduction of behavior. In everyday language, imitation carries with it the implication of intentionality or purposiveness (see Mitchell, 1987). When humans use imitation to describe their own behavior, it implies that there is an understanding of the relation between that behavior and the behavior being modeled (or visually demonstrated). For example, when a child imitates the

Address correspondence to: Thomas R. Zentall, Department of Psychology, University of Kentucky, 202B Kastle Hall, Lexington, KY 40506-0044.

E-mail: Zentall@pop.uky.edu 
behavior of an adult, one assumes that the child understands that the two behaviors match.

According to Freud (1933), imitation forms an integral part of the process of identification, and it is motivated by the need to identify (specifically with the same-sex parent). To Piaget (1955), imitation is a cognitive process reflecting an understanding or assimilation of the relation between one's own body parts and those of others (imagine a human adult walking with his hand clasped behind his back and being followed by a toddler with his hands also clasped behind his back). According to this view, imitation is an intentional, conscious process involving the ability to take the perspective of another (see also Whiten, in press). If this view is correct, and it can be shown that animals are capable of imitative learning, then it follows that animals have a more advanced representational system than many have thought them to have.

But psychologists and biologists who study animals have also been interested in how animals learn from each other (generally members of their own species), without regard for the implications of such learning for the animal's capacity for cognitive processing. These researchers are interested in the more general area of social learning in which learning by an animal is influenced by the presence of or by the experiences of another animal and in how such influences contribute to the animal's fitness. For these researchers, the function of social learning is of more interest than the mechanisms responsible for its occurrence. This often unarticulated distinction has led to a considerable misunderstanding among researchers (see Galef, 1988b).

The purpose of this review is first to classify the various forms of social influence depending on what mechanisms are thought to underlie them (see Table 1). For example, among the simplest kinds of social influence are those social behaviors that are typical of the species and that happen to occur in unison (e.g., schooling, flocking, and herding). These behaviors can be thought of as genetically predisposed. Other behaviors may be influenced by a change in the motivation of the observer, and the mere presence of another animal may increase or decrease the level of the observer's motivation. Still other behaviors may occur because the demonstrator or model can serve as a salient discriminative stimulus that predicts the appearance of food and allows for simple associative learning. Additionally, it is possible for the behavior of a demonstrator to simply draw the observer's attention to a location or to a stimulus that is the focus of the target behavior (e.g., 
Table 1. A taxonomy of social influence and social learning

A. Species Typical Factors (released behavior)

1. Mimicry

2. Contagion
a. Courtship
b. Predator directed
c. Feeding behavior

B. Motivational Factors (induced motivation influences learning)

1. Social facilitation

2. Incentive motivation

3. Observation of aversive conditioning

C. Perceptual Factors (attention getting function)

1. Local enhancement

2. Stimulus enhancement

D. The Nature of the Response

E. Simple Learning Factors (behavior as a discriminative stimulus)

1. Imprinting

2. Following (matched dependent)

3. Observational conditioning

4. Bird song (vocal imitation, stimulus matching)

5. Visual matching

6. Novel behavior

F. Imitative Learning (motor pattern copying)

1. Two-action method

2. Program level imitation

3. Gestural imitation

4. Goal emulation

5. Generalized imitation

6. Intentionality and imitation

7. Symbolic imitation

a bar that must be manipulated to obtain food) and the observer's mere proximity to that location or stimulus may make the target behavior more probable. In many cases, more than one of these social mechanisms may be involved. Finally, when all other forms of social influence and social learning have been ruled out or controlled, whatever remains may be attributable to visual-mediated imitation.

For those interested specifically in imitative learning, its isolation from other simpler mechanisms has not been an easy task (see Heyes, 
1996). Whenever an alternative mechanism is identified, new designs must be conceived that appropriately segregate imitation from alternative accounts. In this review, each of these classes of social influence and social learning in turn will first be examined and then an attempt will be made to try to identify procedures that may separate them from imitative learning.

A second purpose of this review is to clarify the distinction between the function of imitation (and social learning in general) for the survival of the organism and the mechanisms responsible for its occurrence. Finally, an attempt will be made to identify the mechanisms by which organisms are able to imitate.

The terms social influence and social learning extend to any influence that an organism may have on another that results in a similarity of behavior or appearance between the two. The organization of Table 1 attempts to classify the various forms of social influence and social learning according to the mechanisms that underlie them.

Because biologists typically take a genetic approach to the study of organisms, for them, both physical appearance and behavior have evolutionary bases and thus, are closely related. Thus, one starts with physical appearance and behavior that are largely under specific genetic control.

\section{SPECIES TYPICAL FACTORS}

\section{Mimicry}

When imitation involves copying of the physical appearance of one species by another, it is generally referred to as mimicry. When a relatively defenseless animal takes on the appearance of an animal that has better defenses, it is known as Batesian (or Mertensian) mimicry. A well-known case of Batesian mimicry is that of the palatable viceroy butterfly mimicking the appearance of the unpalatable monarch butterfly (Turner, 1984). But of course, such mimicry results from natural selection of the increased fitness incurred by those moths that cannot easily be discriminated by predators from the bad-tasting butterfly.

A special case of mimicry involving behavior is the broken-wing display of certain ground-nesting birds, such as the killdeer or the avocet (Sordahl, 1981). When the female bird is near the nest and a predator approaches, the bird flies away from the nest while mimicking the erratic flight pattern that might be shown by a bird with a broken wing. 
Although one can speculate about the origins of this behavior, it appears to be genetically based (i.e., the predator serves as a releaser; thus, it does not require learning).

\section{Contagion}

When two or more animals engage in similar behavior and that behavior is species-typical, the coordinated behavior is often attributed to contagion (Thorpe, 1963) also called mimesis (Armstrong, 1951) or response facilitation (Byrne, 1994). Contagion can be used to describe certain courtship displays when they involve coordinated movements between the male and female that can sometimes appear to be virtual mirror images (Tinbergen, 1960). Also, antipredatory behavior can be considered contagious when it involves the coordinated movement of a group of animals for defensive purposes. Such behavior occurs in certain mammalian (herding) and avian (flocking) species. When this coordinated behavior is aggressive (i.e., directed toward rather than away from danger), it is known as mobbing (Hoogland \& Sherman, 1976). Contagion can also be shown in an appetitive context. A satiated animal in the presence of food will often resume eating upon the introduction of a hungry animal which begins eating (Tolman, 1964). In the case of contagion, the behavior of one animal appears to serve as a releaser for the unlearned behavior of others (Thorpe, 1963).

\section{MOTIVATIONAL FACTORS}

When an attempt is made to assess imitative behavior, an observing animal (e.g., a rat) is exposed to a demonstrator (rat) that is performing a novel response (such as bar pressing) and the facilitated rate of acquisition of the novel response is taken as a measure of imitation. But because the rate of acquisition is a relative measure (it will depend on a variety of procedural factors unrelated to the conditions of observation), it must be compared with that of a control group. The choice of the control group often depends on the way the experimental questions are asked. For example, early research appeared to assume that rats could learn through observation of the performance of a demonstrator, and the question was, did such learning occur more quickly than the more typical shaping procedures (i.e., by successive approximations) (Corson, 1967; Jacoby \& Dawson, 1969; Powell, 1968; 
Powell \& Burns, 1970; Powell, Saunders, \& Thompson, 1969). The experimental question asked in these early experiments appears to have been a practical one: Would observation of the demonstration of a response be a faster means of training rats to bar press? The problem with the use of a "shaped" control group is that the procedures used to shape animals to bar press are difficult to specify in ways that can be accurately reproduced. Furthermore, comparison of the rate of bar-press acquisition by an imitation group with that of a group of shaped animals may indicate little about the ability of animals to imitate, especially if the imitation group acquires the response at the same rate as, or slower than, the control group. To ask about the ability of animals to acquire a response through observation of a demonstrator, a more appropriate control group would be a "trial-and-error" control (i.e., a group of animals that acquires the response on its own).

\section{Social Facilitation}

The implication of imitative learning is that information transmitted from the demonstrator to the observer has led to facilitated acquisition. There is evidence, however, that the mere presence of an animal (of the same species, i.e., a conspecific) can have effects on the motivational state of an observer (Zajonc, 1965), and that a change in the observer's motivational state can affect the acquisition and performance of a response (Levine \& Zentall, 1974; Zentall \& Levine, 1972). Zajonc has referred to this effect of the "mere presence" of a conspecific on the observer's motivation as social facilitation, and he has proposed a version of Hull's (1943) theory to account for the variety of findings of behavioral facilitation and inhibition that have been reported in humans and animals. According to Zajonc, the presence of a conspecific leads to an increase in arousal, which can actually lead to the retardation of acquisition of a novel (to-be-learned) response. Others have suggested that the mere presence of a conspecific may facilitate the acquisition of a new response for the same reason (Gardner \& Engel, 1971) or because the conspecific may have the ability to reduce fear in the observer (Davitz \& Mason, 1955; Morrison \& Hill, 1967). In any case, comparison of acquisition of an observed behavior with acquisition by trial-and-error in not sufficient. Experiments concerned with true imitative learning must include a control for the possibility of facilitation 
(or retardation) of response acquisition resulting from the mere presence of a conspecific.

One further potential source of demonstrator-provided motivation should be mentioned. Although the mere presence of a conspecific may contribute to the motivational state of an observer, the general (nonspecific) activity of the demonstrator may make an additional contribution. Being in the presence of an active conspecific (i.e., one that is working for food but that is responding in a way that is irrelevant to the target response) might constitute an even better control than mere presence. This point will be returned to later.

\section{Incentive Motivation}

Reinforcement provided to the observer during the demonstration of a novel response (i.e., incentive motivation) may also play a role in the rate at which a novel response is acquired. Although Del Russo (1971) did not find significant evidence for imitative learning by rats that observed a demonstrator bar pressing for food (relative to a trial-and-error control), he did find significant facilitation of bar pressing by a group of observers that got fed whenever their bar-pressing demonstrators got fed. This facilitation may have involved a general increase in arousal on the part of the reinforced observer or a more specific association of the apparatus context with reinforcement. In either case, observers that receive reinforcement when demonstrators make the appropriate response would likely be more active following observation than nonreinforced comparison groups, and more active animals would be more likely to make accidental contact with the bar.

\section{Observation of Aversive Conditioning}

Observation of a novel response being acquired (or being performed) by a demonstrator that is motivated by the avoidance of painful stimulation (e.g., electric shock) may be a particularly good procedure to use in searching for evidence of imitative learning because of the great evolutionary value that such social learning should have (i.e., learning from the mistakes of others). However, the observation of a demonstrator in distress presents the need for a special kind of control. Emotional cues provided by a conspecific either escaping from or avoiding shock, may provide emotional cues of pain or fear that could instill fear in an 
observer. For example, John, Chesler, Bartlett, and Victor (1968) found that cats which had observed a demonstrator being trained to jump over a hurdle to avoid foot shock, learned the hurdle-jumping response faster than controls that did not observe the demonstrators. It may be, however, that being in the presence of a cat being shocked was sufficient to increase the observers' fear (motivation) associated with the conditioning context. Under such conditions, a change in motivation may facilitate acquisition.

Under different conditions, however, although rats that observed a trained demonstrator which had acquired a discriminated shuttle avoidance response acquired that response faster than rats that observed a merely present demonstrator; rats merely exposed to the empty shuttle box acquired the shuttle response fastest (Sanavio \& Savardi, 1980). Thus, trying to sort out mere presence and emotional motivational effects from learning effects can be quite complex. Although using well-trained demonstrators can reduce the likelihood of pain-produced cues being transmitted to the observers (Del Russo, 1975), it may not be possible to avoid the effects of demonstrator-provided, fear-produced cues.

One way to avoid problems associated with differential motivational cues encountered with observation of aversively motivated conditioning is to include a control group that is exposed to performing demonstrators but with the observer's view of a critical component of the demonstrator's response blocked. Such a control was included in an experiment by Bunch and Zentall (1980), who used a candle flame avoidance task originally developed by Lore, Blanc, and Suedfeld (1971). Laboratory rats show a natural curiosity when presented with a candle flame. They cautiously approach the flame and withdraw quickly on contact (when the flame contacts their nose and singes their whiskers). Even so, repeated contacts are made until they learn to avoid contact with the flame. Bunch and Zentall found that rats learned the candleflame-avoidance task faster after having seen a demonstrator acquire the task, as compared with (1) a group for which a small barrier was placed in front of the candle such that the observer's view of the rat's contact with the candle was blocked, and (2) a social facilitation control group. Thus, although a variety of auditory cues (a potential by-product of the demonstrator's pain), olfactory cues (e.g., potentially produced by singed whiskers, defecation, and urination), and visual cues (e.g., seeing the demonstrator approach and then rapidly withdraw from 
something directly behind the barrier) associated with the task should have provided comparable motivational cues to these control observers, task acquisition was not facilitated as much as for observers could also observe the demonstrator's contact with the candle.

Another means of controlling for potential motivational cues provided by the demonstrator performing a pain- or fear-motivated task, is to expose the observers to demonstrators performing a discrimination (Kohn, 1976; Kohn \& Dennis, 1972). In this research, rats that observed a demonstrator performing a relevant shock-avoidance discrimination acquired that task faster than controls for which the demonstrator's discrimination was the reverse of the observer's (i.e, what was correct for the demonstrator was incorrect for the observer and vice versa). However, although it may be possible to control for the social transmission of motivation produced during the acquisition or performance of an avoidance response, it is also possible that general fear induction (from the demonstrator to the observer) may decrease the likelihood of finding evidence for imitative learning in such a context.

\section{PERCEPTUAL FACTORS}

When the observation of a demonstrator draws attention to the location or the object of an action (e.g., a lever), it may merely alter the salience of the lever (stimulus enhancement) or the place in the environment where the lever is located (local enhancement). Thus, whenever the behavior being demonstrated is directed towards an object, that object may attract the observer's attention independently of what the demonstrator is doing to that object (i.e., the behavior to be imitated). In fact, animals may learn much about the functional significance of objects in nature by having their attention drawn to those objects when manipulated by conspecifics. But learning facilitated by such attention-getting would not qualify as imitation.

\section{Local Enhancement}

Local enhancement refers to the facilitation of learning that results from drawing attention to a locale or place associated with reinforcement (Roberts, 1941). For example, Lorenz (1935) noted that ducks enclosed in a pen may not react to a hole large enough for them to escape unless they happen to be near another duck as it is escaping from the pen. 
The sight of a duck passing through the hole in the pen may simply draw attention to the hole.

Local enhancement has also been implicated in the finding that puncturing the top of milk bottles by great tits will spread in a systematic way from one neighborhood to another (Fisher \& Hinde, 1949). Although the technique of pecking through the top of the bottle may be learned through observation, it is also likely that attention was drawn to the bottles by the presence of the feeding birds, and once at the bottles, the observers found the reward and consumed it. Then, learning to identify milk bottles as a source of food, can readily generalize to other open bottles, and drinking from opened bottles can readily generalize to an attempt to drink from a sealed bottle, which in turn can lead to trial-and-error puncturing of the top. Although quite speculative, such a sequence of simpler mechanisms does a good job of accounting for the spread of this ingenious behavior.

As Denny, Clos, and Bell (1988) have shown, local enhancement can be studied in its own right. Exposing rats to the movement and sound of a bar being activated (by the experimenter from outside the chamber) and followed by the presentation of food can facilitate the acquisition of the bar-press response by the observers, relative to various control procedures.

Local enhancement may also account for John et al.'s (1968, Exp. 2) finding of socially facilitated acquisition of lever pressing by cats. Cats in the experimental group that observerd another cat-lever pressing for food, learned to press that lever faster than cats in the control group who observed another cat who was fed periodically without lever pressing. But observation of lever pressing may simply draw attention to the lever. Local enhancement is especially likely in this context, in which observation of the moving lever might encourage lever approach upon removal of the demonstrator (especially by cats, a species known for its motivation to explore).

Similarly, local enhancement may play a role in the faster acquisition of lever pressing by kittens that observed their mothers as demonstrators, than by kittens that observed an unfamiliar female demonstrator (Chesler, 1969), because orientation towards the mother may be more likely than orientation towards an unfamiliar cat.

Local enhancement may also be involved in John et al.'s (1968, Exp. 1) finding of facilitated acquisition of an aversively-motivated hurdle-jump response. The distinction between imitation and local 
enhancement may be a subtle one in this case, but observation of the demonstrator may simply draw the observer's attention to the top of the hurdle. In other words, seeing a ball bounce over the hurdle, or even placing a flashing light at the top of the hurdle might be enough to facilitate the hurdle-jumping response. In general, whenever the performance observed involves an object (i.e., a manipulandum) to which the observer must later respond, local enhancement may play a role (Corson, 1967; Denny et al., 1988; Herbert \& Harsh, 1944; Jacoby \& Dawson, 1969; Oldfield-Box, 1970).

In other cases, it may be possible to control for local enhancement effects by including proper controls. Lefebvre and Palameta (1988), for example, found that pigeons who observed a model pierce the paper cover of a food well to obtain hidden grain, later acquired that response on their own, whereas those that observed that same response, but with no grain in the well (the model performed in extinction), failed to acquire the response.

\section{Stimulus Enhancement}

In the case of local enhancement, the attention of an observer is drawn to a particular place by the activity of the demonstrator. The term stimulus enhancement is used when the activity of the demonstrator draws the attention of the observer to a particular object (e.g., the manipulandum). Quite often in the study of imitative learning, the object in question is at a fixed location so local enhancement and stimulus enhancement are indistinguishable. In the duplicate-chamber procedure (see Warden \& Jackson, 1935; Gardner \& Engel, 1971), however, there are two manipulanda (e.g., levers), one in the demonstration chamber and the other in the observation chamber. Under these conditions, drawing attention to the demonstrator's lever should not facilitate acquisition of lever pressing by the observer. In fact, one could argue that it should retard acquisition of lever pressing by an observer because it should draw the observer's attention away from its own lever. However, the similarity between the demonstrator's lever and that of the observer may make it more likely that the observer notices its own lever after having its attention drawn to the demonstrator's lever (Henning \& Zentall, 1981; Levine \& Zentall, 1974; Zentall \& Levine, 1972). Thus, stimulus enhancement refers to the combination of a perceptual, attention-getting process resulting from the activity of the demonstrator 
in the presence of the lever, and stimulus generalization between the demonstrator's lever and the observer's lever. Because it subsumes local enhancement, the term stimulus enhancement may be more inclusive and thus, may be preferable (Galef, 1988b).

Stimulus enhancement may also be involved in the facilitated acquisition by an observer of a demonstrator performing a discrimination (e.g., responding in the presence of $\mathrm{S}+$ but not in the presence of $\mathrm{S}-$ ). If the demonstrator is required to make contact with the positive stimulus, but not the negative stimulus, the positive stimulus is likely to attract the observers attention and responding to it may be facilitated (Edwards, Hogan, \& Zentall, 1980; Kohn, 1976; Kohn \& Dennis, 1972; Fiorito \& Scotto, 1992; Vanayan, Robertson, \& Biederman, 1985).

Stimulus enhancement may also play an important role in mate-choice copying by guppies (Dugatkin, 1996). Female guppies that see a model female in the presence of a courting male will tend to prefer that male over an alternative male (Dugatkin, 1992; Dugatkin \& Godin, 1992). But the courting behavior itself may draw attention to the male, and the observing female may be more attracted to the familiar male than to the unfamiliar male.

The facilitation of learning through perceptual factors presents a most difficult problem for the study of imitation in animals. If the similarity between the demonstrator's location or manipulandum and that of the observer presents an interpretational problem because of perceptual factors, making the location or the nature of the manipulandum for the observer different from that of the demonstrator is likely to interfere with the observers "understanding" of the relation between the two tasks. This problem, which will be addressed later, will require a new approach to defining adequate control procedures.

\section{THE NATURE OF THE RESPONSE}

Earlier it was suggested that in order for a response to be considered acquired through imitation it must be novel (Thorpe, 1963). This stipulation is useful in helping to distinguish cases of imitation from behavior already in an animals repertoire, the probability of which may increase due to other factors (e.g., species typical releasers, increased motivation resulting from the presence of a conspecific, or attention-getting aspects of the demonstrator's behavior). But a clear adequate definition of novel response is not easy to provide and thus, 
this requirement may not be useful. First, to ensure that the response is not already in the observer's repertoire, one must have a complete record of the animals past experience. Second, because one can argue that any behavior that one defines as novel is actually similar to some behavior already in the animals repertoire (e.g., pressing a bar may be considered similar to climbing or stepping onto an object or similar to manipulating a piece of lab chow).

Novel, in the sense that it is used in the present article and in much of the literature, is used empirically and by exclusion. Thus, if the probability of the behavior is low at the start of the experiment and an increase in the behavior occurs that cannot be attributed solely to the introduction of a releaser, an increase in motivation produced by the presence of a conspecific, or to attention drawn to a manipulandum (or to a location or stimulus), then the behavior can be thought of as novel (see Huber, 1998).

\section{SIMPLE LEARNING FACTORS}

There are a number of cases of social learning which may be mediated by simple nonsocial learning mechanisms. Although social stimuli are present and those social stimuli may play a role in facilitating acquisition of the target behavior (perhaps because the social stimuli are more salient than nonsocial alternatives), the mechanisms by which the observer acquires the behavior may be more parsimoniously explained in terms of simpler species-typical or individual learning processes.

\section{Imprinting}

The first example of social learning that should be distinguished from true imitation is imprinting. Imprinting is a process that occurs primarily in species that do not have the luxury of a nest or den in which to protect their young. In such species (e.g., fowl and grazing mammals), the young are hatched (or born) in a precocious state that allows them to move about following a very brief period of inactivity. To compensate for their mobility (which could also put them at great risk of predation) most of these species have evolved the predisposition to follow the first moving object they see. Although this object is generally their mother, laboratory experiments show that almost any moving object can function as the object of imprinting (Hess, 1973). 
Imprinting is a curious process that combines a strongly predisposed behavior (following) with considerable flexibility (learning) in the nature of the object that is followed. Although one could say, in a very general sense, that the imprinted young are imitating the mother, the act of following (or approach), is more parsimoniously interpreted as a simple conditioning process, with fear reduction serving as the reinforcer (Kovach \& Hess, 1963).

\section{Discriminated Following (or Matched-Dependent) Behavior}

Rats can learn to follow a trained conspecific to food in a $\mathbf{T}$ maze (if following is rewarded) in the absence of any other discriminative stimulus (Bayroff \& Lard, 1941; Church, 1957; Haruki \& Tsuzuki, 1967). This kind of learning is sometimes referred to as discriminated following or matched-dependent behavior. Although the leader rat in these experiments is clearly a social stimulus, the data are more parsimoniously interpreted in terms of simple discriminative learning. If, for example, the demonstrator or leader were replaced with a block of wood pulled along by a string, or even an arrow at the choice point directing the rat to turn left or right, one would refer to the cue (i.e., the demonstrator, the block of wood, and the arrow) as a simple discriminative stimulus. Even if following a demonstrator leads to faster learning than following a passive signal, it might merely indicate that the social cue was more salient than either a static or a moving nonliving cue (see Stimbert, 1970). For matched-dependent behavior to be analogous to imitation, the untrained animal would have to follow the demonstrator on the first trial. Even then, however, the motivation to affiliate could account for the following behavior.

\section{Observational conditioning}

The observation of a performing demonstrator may not merely draw attention to the object being manipulated (e.g., the lever), but because the lever press is paired with a consequence to the demonstrator, a Pavlovian association may be established (Zentall \& Levine, 1972) (see also Zentall \& Hogan (1976)). This form of conditioning has been called observational conditioning (Zentall, 1996), valence transformation (Hogan, 1988), emulation, or affordance (Tomasello, 1990). In this case, the observer learns the relation between some part of the enviromnent 
and the reinforcer (e.g., that the top of a box can be removed to reveal what is inside). Similarly, in the case of aversive consequences, the pairing of an object with the demonstrator's fear response can lead to observational conditioning (Mineka \& Cook, 1988; Whiten \& Ham, 1992).

Although observational conditioning would have to take the form of higher-order conditioning (because the observer would not actually experience the unconditional stimulus), there is evidence that such higher-order conditioning can occur in the absence of a demonstrator. If, for example, the onset of a localizable light is followed shortly by the presentation of inaccessible grain, it is sufficient to produce pecking by pigeons to the light (Zentall \& Hogan, 1975). The presence of a demonstrator drawing additional attention to the object to be manipulated (by pecking) and to the reinforcer (by eating) may further enhance associative processes in the absence of imitative learning.

If such a conditioning process is involved, it would suggest that observation of reinforcement of the demonstrator's response should play an important role in such learning. In fact, if the demonstrator's response is not reinforced, or if it cannot be observed, there is evidence that acquisition is impaired (Akins \& Zentall, 1998; Groesbeck \& Duerfeldt, 1971; Heyes, Jaldow, \& Dawson, 1994). Furthermore, rats appear to acquire a bar-pressing response faster following observation of a bar-pressing demonstrator if they are fed at the same time as the performing demonstrator (Del Russo, 1971). Although that result was mentioned earlier in the context of increased motivation on the part of the observer, it is also possible that feeding the observer following the demonstrator's response may result in simple Pavlovian conditioning (i.e., the pairing of bar movement with food).

Observational conditioning may also play a role in an experiment in which observation of experienced demonstrators facilitated the opening of hickory nuts by red squirrels, relative to trial-and-error learning (Weigle \& Hanson, 1980). Differential local enhancement can be ruled out, in this case, because animals in both groups quickly approached and handled the nuts, and the observers actually handled the nuts less than controls (perhaps because observers were more efficient at opening them). However, observers alone got to see the open nuts and they had the opportunity to associate open nuts with eating by the demonstrator. 
Similarly, in a experiment already described, Palameta and Lefebvre (1985) showed that a majority of pigeons in three experimental groups that observed a demonstrator piercing a paper cover to obtain grain were able to show similar paper-piercing behavior after a single period of observation, whereas pigeons that observed the demonstrator eating without having to pierce paper and those that observed paper-piercing with no food to eat, in general did not learn. Although this experiment involved several important control conditions, the observers in the experimental groups were the only ones that observed both paper-piercing and food. Thus, these groups were the only ones that observed food that was initially hidden underneath the paper and such learning may confound imitation with affordance or observational conditioning.

Socially transmitted food preferences (e.g., Galef, 1988a; Strupp \& Levitsky, 1984) may represent a special case of observational conditioning. Although food preference would appear to fall into the category of unlearned behavior subject to elicitation through contagion, consuming a food with a novel taste can be thought of as an acquired behavior. The mechanisms responsible for socially acquired food preferences have been posited to have simple associative learning components (e.g., learned safety or the habituation of neophobia to the novel taste), for which the presence of a conspecific may serve as a catalyst (see Galef, 1996). On the other hand, there is also evidence that learning phenomena (e.g., blocking, overshadowing, and latent inhibition) readily found conditioning experiments with more typical stimuli and outcomes, are not easily found in experiments involving the social enhancement of food preferences (Galef \& Durlach, 1993). Thus, different mechanisms may be involved.

One of the best examples of observational conditioning is in the acquisition of fear of snakes by laboratory-reared monkeys exposed to a wild-born conspecific in the presence of a snake (Mineka \& Cook, 1988). Presumably, the fearful conspecific serves as the unconditioned stimulus and the snake serves as the conditioned stimulus. In support of this hypothesis, it appears that exposure to a fearful conspecific alone or to a snake alone is insufficient to produce fear of snakes in the observer. 


\section{Bird Song}

A special case of matching behavior by animals is the acquisition of bird song (Hinde, 1969; Marler, 1970; Nottebohm, 1970; Thorpe, 1961) (see also vocal mimicry, e.g., Pepperberg (1986) and Thorpe (1967)). Although for many species of songbird the development of species-typical song is regulated to a large extent by maturation and the seasonally fluctuating release of hormones, regional variations in the song appear to depend on the bird's early experience with conspecifics (Baptista \& Petrinovitch, 1984). Thus, one could say that young songbirds learn their regional dialect by imitating the song of more mature conspecifics.

Acquisition of bird-song dialect is a special case of imitation for three reasons. First, although it is learned, bird song is a variation on a species-typical behavior and thus, is relatively constrained. Second, according to Heyes (1994), in the acquisition of bird song, components of the matching behavior occur by chance and these components increase in frequency because they are intrinsically rewarding. Heyes refers to such behavior as copying rather than imitation. But finally, and most importantly, bird song takes place in the auditory modality. A characteristic of auditory events is that the stimulus produced by the demonstrator and that produced by the observer (or more accurately, the listener) can be a close match, not only to a third party (i.e., the experimenter) but also to the observer. Thus, verbal behavior, for which comparisons between one's own behavior and that of others is relatively easy because one can hear one's own utterances with relative fidelity, may be a special "prepared" case of generalized, stimulus identity learning (e.g., pigeons that have been trained to match shapes a pear to be able to apply the principle of matching to novel hue stimuli; see Zentall, Edwards, and Hogan (1983)).

This analysis of the imitation of verbal behavior can also be applied to certain examples of visual imitation. Any behavior that produces a clear change in the environment, such that from the perspective of the observer there is a match between the stimulus produced by the demonstrator and that produced by the observer, may be a case of stimulus matching (e.g., observing someone turning up the volume of a radio - when the knob turns to the right, the volume increases). Such cases of visual stimulus matching can be distinguished from the more abstract and interesting case in which no visual stimulus match is poss- 
ible (e.g., the imitation of a person who has his hands clasped behind his back).

The preceding sections outline the various conditions under which the behavior of animals can be influenced by the presence and behavior of others. In each case, it has been argued that the change in the observer's behavior cannot be unambiguously attributed to imitative learning. But this emphasis on the importance of imitation should not detract from the value of these other mechanisms, particularly their functional value to the fitness of the observer. In fact, taken together, the many ways in which the behavior of conspecifics can influence the behavior of observers suggest the importance of a variety of social cues in the survival of many animal species.

\section{Response novelty}

To this point it has been assumed that the to-be-imitated response is novell, that is, the response is not already in the repertoire of the observer. In fact, however, one can argue that no response can be truly novel because any species can do only a limited number of actions and most basic motor patterns will have been made prior to the experiment (see Whiten \& Custance, 1996). On the other hand, although all birds have pecked at objects, it is not likely that they have pecked at the particular object (e.g., a floor treadle) that they observed another bird pecking. Thus, other things being controlled for, why should a pigeon peck at that object rather than at other objects. In fact, why should a pigeon peck at the treadle rather than make some other response (e.g., step on it). A reasonable criterion would be that the response should be novel under the conditions of test. That is, the response should have a very low probability of being made by the observer in the absence of demonstration and under conditions that control for the other nonimitative factors already discussed.

\section{IMITATION}

True imitation has been defined as "the copying of a novel or otherwise improbable act or utterance, or some act for which there is clearly no instinctive tendency" (Thorpe, 1963, p. 135). The preceding analysis allows one to be more precise. 
- First, for true imitation to be demonstrated, the target behavior should not already be part of the observing animal's repertoire-whether improbable or not (Clayton, 1978).

- Second, one should control for motivational effects on the observer produced either by the mere presence of the demonstrator or by the mere consequences of the behavior of the demonstrator.

- Third, one should control for the possibility that the demonstrator's manipulation of an object merely draws the observer's attention to that object (or one like it), thus making the observer's manipulation of the object more probable.

- And finally, one should control for the simple pairing of a novel stimulus (e.g., a lit response key or the movement of a bar) with the presentation of inaccessible food).

Some have argued that true imitation requires that the observer recognize the intentional structure of the actions of the demonstrator (Tomasello, Kruger, \& Ratner, 1993), but if this level of evidence is required then imitation is not likely to be found in any nonverbal animal and many examples of imitation in young humans with some language ability would have to be rejected.

In much of the discussion that follows, data are reported from species other than primates. This should not be taken to suggest that primates, especially monkeys, are less capable of leaming through imitation (though such an argument has been made; see Fragaszy and Visalberghi $(1989,1990))$. Imitative learning by primates has often been studied under quasi-natural conditions that do not lend themselves to the kind of rigorous analysis has been fostered here. In many cases, it is suspected that true imitative learning plays an important role in many primate studies on social learning, but it is simply not possible to come to such a conclusion because in most cases control for all of those alternative accounts based on social influence and nonimitative social learning has not even been attempted.

\section{The Two-Action Method}

Earlier, it was noted that the control of perceptual factors may prove to be difficult because they are related to the similarity between the observer's and demonstrator's behavior, and they should be similar if one expects imitation to occur. One way to deal with all of the problems of control is to keep all extraneous factors constant, between groups, 
and manipulate only the critical defining feature, the topography of the to-be-imitated response.

Custance, Whiten, and Fredman, (1999) used "artificial fruit" to simulate the shell of fruit that must be removed by monkeys to gain access to the edible portion inside. In fact, demonstrators opened a latched clear plastic box in one of two distinctive ways. Observer monkeys showed a significant tendency to open the box by the same means as they observed it demonstrated.

Similarly, Dawson and Foss (1965), found that budgerigars acquired a lid-removal task (by trial-and-error) in one of three different ways: Pushing the lid off with the beak, twisting it off with the beak, or grasping it with the foot and pulling it off. Observers were then exposed to these performing birds, and when they were then given the opportunity to perform themselves, each observer removed the lid in same manner as its demonstrator (see also, Galef, Manzig, and Field (1986) for more modest results with this procedure).

Will, Pallaud, Soczka, and Manikowski (1974) noted a related effect in a study in which rats observed either a trained demonstrator performing a successive discrimination or an experimentally naive demonstrator. They found that the trained demonstrators typically responded with one of three distinctive patterns when the discriminative stimulus was available and that the observers learned not only to respond in the presence of one stimulus and not in the presence of the other, but they also learned the pattern of responding of their demonstrator (e.g., alternating a bar press with eating or making a burst of bar presses followed by eating the accumulated pellets).

Heyes and Dawson (1990) have reported similar results by rats that observed demonstrators expressly trained to respond in one of two different ways. After observing demonstrators push an overhead bar either to the left or to the right, Heyes and Dawson found that observers given access to the bar tended to push the bar in the same direction as their demonstrator. Remarkably, the observers matched the demonstrators' behavior in spite of the fact that, because the observers faced the demonstrators during the period of observation, the direction of bar motion (relative to the observer's body) during observation was opposite that of the bar's motion when the observers performed.

In principle, the two-action method provides the best control for all of the nonimitational factors mentioned earlier. In practice, however, in much of the research that has used the two-action method, in addition 
to the differences in response topography between the observation conditions there have been differences in the consequences of those topographies. For example, in the budgerigar lid-removal experiment, one of the demonstrators learned to push the lid back, whereas another demonstrator learned to grasp the lid in its beak and twist it off (as if it were hinged at one side-edge). Thus, each of the different response topographies found by Dawson and Foss (1965) had a different effect on the lid. To what extent did the distinctive movement of the lid (lids that slide off to the back versus lids that twist off to the right), rather than the demonstrator's response topography, produce the strong correlation between the observer and demonstrator lid removal technique? The birds may have learned how lids work, or what developmental psychologists sometimes refer to as object movement reenactment or affordance (Gibson, 1979; Tomasello, 1996; Whiten, 1998) rather than the appropriate response for a bird to remove the lid. Similarly, in the artificial fruit experiment (Custance et al., 1998; Whiten et al., 1996), seeing the way in which the latch worked (independent of the responsible topography or morph used by the demonstrator) may have contributed to the matching behavior.

In the Heyes and Dawson (1990) experiment, too, the overhead bar moved in different directions (toward different walls of the demonstrator's chamber) for the two observation groups. It is unlikely that the movement of the bar to a particular location was solely responsible for the reported effect; however, because Heyes et al. (1994, Experiment 2) reported similar effects when the bar was moved between the time of observation and observer performance from the common wall between the two chambers to one of the side walls (i.e., a $90^{\circ}$ shift in the direction of possible movement of the bar). However, it is also possible that olfactory cues, specific to the side of the bar against which the demonstrators pushed, was responsible for this imitation-like effect (Gardner \& Heyes, 1998; Mitchell, Heyes, \& Dawson, 1999; Ray \& Heyes, 1998).

Many recent experiments that use the two-action method also fail to control for the difference in affordance provided by the opportunity to observe. Campbell, Heyes, and Goldsmith (1999) allowed starlings to observe a demonstrator removing a plug from a food container either by pulling it up by a loop or by pushing it down. Although the ability of these birds to learn how the plug can move is of some importance, the relevance of seeing the demonstrator remove the plug cannot be con- 
cluded from this experiment. Other recent research has involved the demonstration of one way to obtain food-generally the harder to two ways (Bunyar and Huber (1999) with marmosets; Fritz and Kotraschal (1999) with ravens). When given access to the apparatus, some of the observers then responded as it was demonstrated, whereas all of the control animals obtained the food by the other, simpler, means. Again, the observers may have responded differently because they were the only ones who were shown how the mechanism worked (i.e., an affordance of the apparatus).

In a variation on the two-action procedure, Akins \& Zentall (1996) tried to overcome the problem of differential environmental consequences by training quail to respond to a treadle for food either by pecking at the treadle or by stepping on the treadle. With a common manipulandum and the common movement of the manipulandum, the effect of the two response topographies on the environment should be common as well. Akins and Zentall found that observers showed a significant tendency to respond to the treadle with the same part of the body (beak or foot) as their respective demonstrator (see Zentall, Sutton, and Sherburne (1996) for similar results with pigeons). Kaiser, Zentall, and Galef (1997) have noted that the two-action method, together with a control for trial-and-error learning, provides the most convincing evidence yet for true imitative learning in animals.

Two important points should be made about this procedure. First, the environmental consequences of stepping and pecking were essentially the same (i.e., everything was the same except the two response topographies). And second, it is very unlikely that there was any similarity between the visual stimulus seen by the observer during observation and that seen by the observer during its own performance of the same response. Specifically, the demonstrator's beak on the treadle must have appeared quite different to the observer from the observer's own beak on the treadle. Similarly, though perhaps not so obviously, when the quail stepped on the treadle (located near the corner of the chamber), they pulled their head back and thrust their head forward. Thus, they could not see their foot making contact with the treadle. Once again, to the observer, the demonstrator's response to the treadle must have appeared quite different from the observer's own response to the treadle. Therefore, any account of the imitation found in these experiments in terms of stimulus matching is quite implausible. 


\section{Gestural Imitation}

A form of imitative learning conceptually related to the two-action method occurs when the gestures of a model are copied. Imitation of gestures has been found in chimpanzees (Custance, Whiten, \& Bard, 1995; Hayes \& Hayes, 1952), dolphins (Harley, Xitco, Roitblat, \& Herman, 1998; Xitco, Harley, \& Brill, 1998), and a parrot (Moore, 1992). Remarkably, especially in the case of the dolphin and the parrot, the models were human rather than a conspecific. Thus there was little similarity between corresponding body parts of the observer and the demonstrator. Because objects were not involved, local and stimulus enhancement should be irrelevant. Furthermore, each imitated gesture serves as a control for the others because it is the topography of the response that is important. In addition, the broad range of gestures that have been shown to be imitated within a few seconds of demonstration suggests that no account based on differential motivation is likely to play a role.

The remarkable ability of some animals to learn from observing a quite different species if perhaps best exemplified by the work of Pepperberg (1988) with a parrot using the model/rival technique (first reported by Todt (1975)). In the model/rival technique, two human experimenters demonstrate the training of a particular behavior to the parrot, with one human taking the role of the teacher and other the role of the student. Analysis of the various mechanisms that contribute to learning under these model/rival conditions clearly is not possible. Certainly, vocal mimicking of the type already discussed in the section on acquisition of bird song plays a role and observational conditioning may play a role as well, but the examples of gestural initiation already cited (Moore, 1992) together with the remarkable cognitive capacity of these birds (see Pepperberg, 1990) suggests that this species is capable of considerable learning through imitation.

\section{Related Variables}

Reinforcement of the demonstrated response. Earlier it was noted that the pairing of the movement of a manipulandum with a refinforcer could increase the probability of the target behavior because of simple learning effects. Using the two-action method, the role of simple learning effects can be examined directly, rather than as an artifact to be avoided. Akins 
and Zentall (1999) have recently found that the correspondence between observer and demonstrator response topography disappears when the demonstrator's responses are not reinforced.

A cognitive account of this finding is that through observation, the observer learns that there is no positive consequence associated with the demonstrator's response and thus, there is no incentive for making the same response. This interpretion assumes that the lack of correspondence between observer and demonstrator response topography results from a performance decrement rather than from a learning decrement.

A simpler account of this finding suggests that with this preparation, an association between the demonstrator's behavior and reinforcement (observational conditioning), is necessary for true imitation to occur. It does not provide an alternative account of imitative learning, however, because it cannot account for the correspondence between the observer's and demonstrator's response topographies. Instead, reinforcement may act as a catalyst to bring out imitative learning.

Observer motivation. To what extent is it important that the observer be motivated to observe? Will an observer attend to and learn about a behavior that leads to a reward for which the observer is not motivated to work? To answer this question, prefed Japanese quail were allowed to observe demonstrators either stepping on a treadle or pecking at the treadle for food. When later tested under moderate levels of food deprivation, these quail showed no evidence of imitative learning. However, quail that observed while deprived of food and later tested under moderate levels of food deprivation showed excellent imitative learning (Dorrance \& Zentall, 1999). Thus it appears that the relevance of the outcome of the observed behavior to the observer's motivation plays a role in imitative learning.

Enculturation. One of the variables that may play a role in imitative learning by primates appears to be the degree to which the animals have had extensive interactions with humans - what Tomasello (1990) refers to as enculturation. Enculturated chimpanzees and orangutans readily show signs of imitative learning (Russon \& Galdikas, 1993, 1995; Tomasello, Gust, \& Frost,. 1989; Tomasello et al., 1993; Tomasello, Savage-Rumbaugh, \& Kruger, 1993), whereas lab housed/reared chimpanzees typically do not (Whiten \& Custance, 1996). 
Enculturation may produce its effect in a number of ways. First, it could reduce the apes' anxiety during test. Second, it could increases their attentiveness to social cues. Third, it could give them prior reinforced experience imitating (i.e., it could allow them to experience a form of learning to learn). A better understanding of the various components of enculturation might provide important insights into imitation by apes.

\section{Program Level Imitation}

Byrne (1994, 1945) and, more recently, Byrne and Russon (1998) have made a distinction between action level imitation (as in pressing a lever or poking at a bolt) and program level imitation that involves learning of a coordinated sequence of actions leading to reward. Byrne and Russon suggest that program level imitation, such as the complex sequence of picking, folding, and chewing barbed leaves shown by mountain gorillas and transmitted socially to others, demonstrates a higher level of imitative learning than action level imitation, which in most cases can be explained by simpler mechanisms such as stimulus enhancement and observational conditioning. Although the concept of program level imitation may prove to be useful in identifying more advanced forms of imitative learning, Byrne and Russon have not demonstrated either that the mechanism responsible for social transmission was not some form of affordance, or that once the elements of the task were acquired, the sequence could not have been produced by trial and error learning (see Whiten, in press).

\section{Generalized Imitation}

Imitation of a particular response can be thought of as one example of a broad class of imitative behavior. One can then ask if an animal can learn to match any behavior of another "on cue" (i.e., can an animal learn the general concept of imitation and then apply it when asked to do so in a "do-as-I-do" test). Hayes and Hayes (1952) found that a chimpanzee (Viki) learned to respond correctly to the command "Do this!" over a broad class of behavior. More recently, Custance et al. (1995) have replicated this result under more highly controlled conditions. Furthermore, Custance and Bard (1994) using the "do as I do" procedure, have found that actions on parts of the body that cannot 
be seen by the performer were just as readily copied as those that could be seen. The importance of behavior that cannot be seen by the performer (e.g., touching the back of one's head) is that it rules out the possibility that some form of visual stimulus-matching might account for the behavioral match. The establishment of a "do as I do" concept not only verifies that chimpanzees can imitate, but it also demonstrates that they are capable of forming a generalized behavioral-matching concept (i.e., the chimpanzees have acquired an imitation concept). It is likely that this level of imitation is limited to humans and the great apes (Miles, Mitchell, \& Harper, 1996; Tanner \& Byrne, 1999).

\section{Goal Emulation}

Under certain conditions, an observer may attempt to reproduce the results that the model's behavior has achieved by a method other than that used by the demonstrator. Whiten and Ham (1992) have used the term goal emulation to describe this "nonmatching" form of imitation in which an observer may "understand" that a particular observed behavior has certain consequences, but it may also recognize that the goal could be achieved by any one of a class of behaviors. Goal emulation should not be confused with what Tomasello has called emulation, a process similar to affordance in which learning about the results of behavior is sufficient to facilitate acquisition.

Goal emulation is a kind of imitation that is similar to what Mitchell (1987) calls fourth-level imitation. At this level, "the organism is not bound to reproduce the model, but reorganizes the relationship between model and copy to its own ends" (p. 207). Imitation at this level is produced extensively by humans (e.g., children engaged in make-believe play), but Mitchell suggests that it also can be found in animals (see next section on intentionality).

The possibility that true imitation could be present in the absence of a match between the behavior of the demonstrator and the observer raises problems for the assessment of imitative learning beyond those already mentioned. The procedure used by Dawson and Foss (1965) provides a useful example. If a budgerigar observes another removing the lid of a food container with its foot but "decides" that it could accomplish the same result (perhaps more easily) with its beak, the observer's behavior would be scored as nonimitative. Thus, the possibility of goal emulation raises potential problems even when the two-action method 
is used. On the other hand, the possible ambiguity in interpretation of findings resulting from use of the two-action method would be a problem only if one failed to find evidence for behavioral matching.

Furthermore, alternatives to the two-action method are even less appealing because they tend to err on the side of failing to rule out simpler mechanisms. Thus, the two-action method remains a useful, albeit relatively conservative test of true imitative behavior.

\section{Intentionality}

Interest in imitation research can be traced, at least in part, to the assumption that true imitation involves some degree of intentionality. This is certainly the case in many of the higher order forms of imitation, such as the human dancer who repeats the movements of the teacher. Unfortunately, intentionality, because of its indirect nature, can only be inferred, and evidence for it appears most often in the form of anecdote rather than experiment. Ball (1938), for example, noted the case of a young rhesus monkey that, while kept with a kitten, was observed to lap its water in the same way as a cat. Ball noted further that lapping is extremely rare in rhesus monkeys.

Similarly, Mitchell (1987), in an analysis of various levels of imitation, provides a number of examples of imitation at these higher levels. For example, he discusses the young female rhesus monkey, who seeing her mother carrying a sibling, walks around carrying a coconut shell at a same location on her own body (Breuggeman, 1973).

Such anecdotes, by their very nature, are selected and difficult to verify. If there were some way to bring these examples of intentional imitation under experimental control, it would greatly increase their credibility. Furthermore, intentionality is sometimes used to indicate not just goal-directed behavior but a mental state involving belief or desire. As there is no way that such mental processes can be verified (even indirectly), such speculation should be avoided.

\section{Symbolic Imitation}

In the highest level of imitative behavior, what Mitchell (1987) refers to as fifth-level imitation, not only does the behavior of the observer not match that of the demonstrator, but the differences are explicit and they are produced for the purpose of drawing attention to certain character- 
istics of the model. Examples of such symbolic imitation can be found in the human use of parody and caricature. Such forms of imitation are mentioned primarily for completeness and to note the degree of subtlety that can be involved in imitation.

\section{Cultural Variation}

One more issue with implications for imitation is that of cultural variation or tradition (Huffman, 1996). When one sees a particular pattern of behavior in one community of human, but not in others, it is generally attributed to culture. When similar variance occurs in chimpanzees and one cannot attribute that variance to enviromnental peculiarities (e.g., the presence of a particular predator), one can also consider it cultural in nature. Presumably, if many members of a community have acquired a particular behavior that is rarely seen in other communities, the within-community spread of the behavior can be attributed to social learning (Whiten \& Ham, 1992) and when this pattern of tradition occurs often in different communities it is easy to draw an analogy to human social groups (Whiten et al., 1998).

As these behaviors are transmitted from one member of the community to others under natural conditions, it is likely that a complex combination of social learning and individual learning mechanisms including imitation are involved. The contribution of this line of research is not in isolating the mechanism responsible for the spread of a novel behavior, but in drawing parallels between chimpanzee and human communities (see Tomasello, 1996).

\section{BIOLOGICAL FUNCTION}

Although imitation has been viewed by psychologists as purposive, intentional, and reflective, when imitation is viewed from the perspective of biological function, it can take on very different characteristics. These functional characteristics may account for the broad range of behavior that has been classified as imitative. For example, rather than viewing imitation as a higher form of learning that requires conceptual ability, Boyd and Richerson (1988) have examined it in terms of its potential specific costs and benefits to an organism, as compared with two other evolutionary strategies: species-typical behavior (primarily under genetic 
control) and individual (trial-and-error) learning (primarily under control of the direct consequences of the behavior to the organism).

The benefit of species-typical behavior is its certainty. Birds do not have to rely on trial-and-error learning to build a nest. They are genetically predisposed or programmed to build them. On the other hand, there is a cost to such inflexible behavior. Should the environment change in a way that is inconsistent with an animal's predispositions, there may not be enough flexibility in the system to allow for survival. The giant panda lives almost entirely on bamboo shoots. There has been little competition for this resource and thus, there has been little need for the panda to develop a varied diet. When bamboo is plentiful the panda can thrive. But with the encroachment of human populations, the bamboo forests have shrunk in size and the panda has become an endangered species.

Trial-and-error learning allows an animal to adjust to a changing environment. For example, in an unpredictable environment where ideal foods may not be available, or where other animals may compete for that food, an animal may be predisposed to follow a more general rule. In humans, the preference for sweet food is an example of such a rule. When such categorical rules are insufficient, more arbitrary rules based on individual experience (learning) may be necessary. For example, many animals (including humans) are predisposed to follow the rule, "If one encounters a novel taste, one should eat only a small amount; if one then gets sick, one should stay away from that taste; if one doesn't get sick one should eat more" (see Garcia \& Koelling, 1966). Thus, animals can learn which foods are good to eat and which are not.

But trial-and-error learning has its cost as well. For an animal to learn what not to eat, it must sometimes risk the negative consequences. Rats can learn what foods to eat by trial-and-error but such behavior can result in them getting poisoned. Learning can be beneficial, but it can also be costly. According to Boyd and Richerson (1988), social learning may serve an intermediate role between species-typical behavior and individual trial-and-error learning. If an animal learns from watching another animal, it can benefit from the trial-and-error learning of the other without having to suffer the consequences of errors. For example, if a rat encounters two novel flavors of food, it will prefer the flavor that it sees another rat eat (Galef, 1988a). Although the mechanism by which food preference are acquired socially is much simpler than true imitation, imitative learning may have evolved for similar 
reasons - it allows the experience of one individual to be acquired by others more efficiently than would be the case with trial-and-error learning, yet retaining much of the flexibility of individual learning.

In human societies, culture and tradition are the means of passing on the experiences of group members to the benefit of other members of the group. Individual learning can last a lifetime; social learning can be passed on for many generations but it allows for considerably more flexibility than does genetically predisposed behavior.

A more functional, inductive approach to the study of imitative learning may also provide insights into the conditions under which it would be most likely to occur (see Davis, 1973; Howard \& Keenan, 1993). The relatively asocial laboratory rat or pigeon used in psychological research may not be most appropriate subject for the study of imitation. Instead, animals with better visual acuity than the rat, and which are more social than the pigeon, may be more likely to imitate (e.g., chimpanzees, Custance et al. (1995), parrots, Moore (1992), and even Japanese quail, Akins and Zentall (1996)).

Although I have argued that animals who engage in social learning are likely to benefit by way of increased fitness over those that engage in only species-typical behavior and individual learning, not all species may benefit from imitative learning. Even highly social species that are capable of considerable behavioral plasticity (in the form of individual learning), such as monkeys, may not benefit from imitation because of the nature of resources in their envirownent. For example, they may be more likely to find and eat novel food in the presence of others who are eating (Fragaszy \& Visalberghi, 1996), but they may not benefit from observing how others find and eat novel food. Thus, although imitative learning appears to be maximized in highly social species, it may not be an inevitable consequence of living in social groups. Imitation appears to be widely scattered among species, with humans and great apes being the most prolific imitators, but dolphins and a number of avian species including parrots, pigeons, and Japanese quail show evidence of imitative learning as well. Imitation by a number of bird species, together with the relative absence of imitation in monkeys (Fragaszy \& Visalberghi, 1989, 1990; Whiten \& Ham, 1992; Visalberghi, 1993) (but see also Custance et al. (1998) and Tomasello and Call (1994)), suggests that a high degree of behavioral plasticity and sociality may be neither necessary nor sufficient for the development of imitative learning. 


\section{PSYCHOLOGICAL MECHANISM}

To this point, imitation has been defined as the matching of demonstrator and observer behavior under conditions that control for other, better understood, reflexive, motivational, or simple learning principles. But the means by which animals imitate have not yet been identified, except only vaguely in the introduction. There has been much speculation about the meaning of true imitative learning. Some have described it as a reflective (conscious?) process (Morgan, 1900) or as "the purposive, goal-directed copying of the behavior of one animal by another" (Galef, 1988b, p. 21), whereas others see it as an extension of simple learning principles (Gewirtz, 1969).

\section{Associative Learning Accounts}

The simplest account of imitation has been provided by social learning theorists (Bandura, 1969; Gewirtz, 1969; Miller \& Dollard, 1941). For most social learning theorists, imitation can be explained as a special case of simple instrumental learning. Gewirtz, for example, proposed that initially, when a demonstrator (or model) engages in a particular behavior, the young observer responds in a variety of ways that are unrelated to the behavior being modeled. Occasionally, and only by chance, a correspondence might occur between the behavior of the model and that of the observer. According to Gewirtz, those instances of behavioral correspondence are typically accompanied by reinforcement. For example, a parent may say "daddy" many times to a young child and on those occasions on which it happens to be followed by "dada" spoken by the child, positive reinforcement (perhaps socially, through the parent's excitement and attention) will often be provided. The word "daddy" then comes to serve as a conditioned stimulus that signals the opportunity to obtain reinforcement for emitting the response "dada." Thus, conditioning theory can explain individual cases of response copying, especially when verbal behavior is involved. But every imitated word does not go through such a processes of reinforcement by successive approximation (i.e., trial-and-error shaping).

To account for the extensive use of imitative learning by children, Gewirtz (1969) proposed that copied responses which occur initially through selective reinforcement, come to generalize to other behavior, without the need for additional consistent reinforcement. If 
generalization, as it is used here, is meant to be explanatory, rather than merely descriptive, however, it requires a more complex mechanism than simple associative learning can provide. Stimulus generalization theory (Spence, 1937) is based on the principle of physical stimulus similarity. Specifically, a reinforced response in the presence of a particular stimulus will tend to occur in the presence of other stimuli, to the extent that those other stimuli are physically similar to the training stimulus. But when applied to imitation, how does an infant generalize from repeating the word "daddy" to repeating the quite-different-sounding word "ball'? Furthermore, the concept of generalization refers to the probability of occurrence of the trained response, rather than a matching response. Thus, according to such a conditioning model, the response "daddy" should occur to other stimuli to the extent that they sound like "daddy."

Alternatively, generalized imitation may be related conceptually to identity learning with visual stimuli, but in the case of imitation, it is the matching of behavior rather than stimuli. When Baer, Peterson, and Sherman (1967) trained retarded children to match several behaviors of a model when instructed to, "Do this," they found that the children continued to match in the absence of reinforcement. They proposed that the children had formed a functional stimulus class defined by the correspondence between the stimulus output of the child's behavior and the stimulus output of the model's behavior. Thus, to account for such stimulus/response matching, a child must, at a minimum, have a concept of identity (i.e., the child must understand what it means that two things are the same) (see Zentall, Edwards, and Hogan (1983)) or what Gewirtz (1969) calls a matching-response class. Such an analysis implies processes that go beyond simple learned associations and their generalization. But children do show evidence of identity learning at a very early age (Tyrrell et al. 1993) and pigeons too show the capacity for identity learning (Zentall, et al., 1981).

The copying of verbal behavior may be explained in this way because one can hear one's own utterances with relative fidelity and can compare them directly with those of a model (i.e., stimulus matching). However, such matching of response-produced stimuli to target stimuli cannot account for imitation by a young child when an adult model says, "Do this," as the model places his hands over his eyes. In this type of imitation, from the perspective of the observer, there 
is no match between the stimulus provided by the behavior of the model and that provided by the observer's own behavior.

\section{Cognitive Accounts}

According to Piaget (1955), true imitation involves sensory-motor assimilation. It is the coordination, first, the sensory-motor system of the individual (or the self), which occurs at an early age and then, an appreciation of the similarities between the individual and others. In Piaget's view, this process occurs first through the similarity between the seen body parts of others and the corresponding seen body parts of child. Later, an acquired cross-modal matching process allows the child to understand the correspondence between its own unseen body parts (e.g., the eyes, nose, and head) and those of others. This cross-modal matching process is based on

1. touch (the felt parts of self and others),

2. the correspondence of touch and sight (the felt and seen body parts of others),

3. the inference that because one's own unseen body parts feel like those of others, they must look like those of others (see also Moore, 1996).

Finally, this assimilation results in a schema of the individual (i.e., an image, in our mind's eye, of ourselves). In explaining the development of imitation in children, Guillaume (1971) views imitation as linked to the child's notion of self and he proposes that "imitation enables the child to see himself in the person of another" (p. 207). Thus, according to this view, the mechanism that makes imitation possible is the ability to take the perspective of another.

However, if imitative learning occurs in species as varied as rats, pigeons, and Japanese quail, as it appears to, the responsible mechanism is not likely to be theory, of mind or perspective-taking. But in cases in which stimulus matching is inadequate to account for imitation, some precursor of perspective-taking is likely to be involved.

But how does a pigeon infer the similarity between its own beak (seen only as a gross distortion) and the beak of a demonstrator? Such an inferential process would seem to be beyond the capacity of rats, pigeons, and quail. 


\section{BIOLOGICAL MECHANISM}

Alternatively, Metzoff (1996) has proposed that in the case of human infants, there is an "inbuilt drive to 'act like' their conspecifics" (p. 363). Metzoff bases his conclusion on data suggesting that infants (and even newborns) (Metzoff \& Moore, 1989; Reissland, 1988) imitate a wide range of adult demonstrated gestures, including lip, check, brow, head, and finger movements, as well as emotional expression. Although Jones (1996) suggested that early research on infant imitation involving tongue protrusion may be accounted for more parsimoniously in terms of very early attempts at the oral exploration of objects, the range of imitated gestures, as well as the number of independent reports of such imitation (Metzoff, 1996), suggest that these effects cannot easily be explained away.

The implications of infant imitation are important because if true imitation can occur in newborns, it suggests that the mechanisms responsible for imitation are probably not cognitively based. Clearly, even the most rudimentary cognitive structures involved in perspective-taking would not have had time to develop in newborns.

The data suggest that infants are born with the ability to engage in "a matching-to-target process in which they actively compare the visual information about the seen body movements [of the adult] with the proprioceptive feedback from their own movements in space" (Metzoff 1996, p. 351). Such innate cross-model matching must be quite different from Piaget's experienced-based process. Metzoff's data suggest that infants do not have to learn the correspondence between the behavior of others and their own; they just appear to do so reflexively. According to this view, infants are "prewired" to imitate the behavior of conspecifics. But the mechanism cannot be so general. Generalized imitation is a category of matching behavior that is defined by the third party (e.g., the experimenter). To the imitator, there is no match, especially if learning is not involved. Thus, if imitation (in humans at least) is an innate response, then each demonstrated behavior that is imitated (e.g., tongue protrusion and brow movement) must be a releaser for the same behavior in the infant. Given the wide range of imitated behavior, the list of releasers must be quite long.

It is difficult to imagine the evolution of such an elaborate set of releasers to account for imitation by humans; however, in species for which a more cognitive perspective-taking account seems even less prob- 
able (e.g., quail and pigeons), the existence of such a set of predisposed releasers definitely may be involved.

\section{CONCLUSIONS}

Procedures have now been developed that are capable of separating true imitative learning from other social influences on behavior. Early results indicate that imitative learning can be found in a variety of species. Such findings should not be surprising because social learning, by imitation and otherwise, provides clear benefits to many organisms over genetically based behavior and trial-and-error learning. The mechanism by which animals are able to match their behavior to that of a demonstrator may involve some form of coordination of visual and tactile sensory modalities (see Mitchell, 1993) and in some species such coordination may be predisposed. However, a more complete account of these processes will have to await research to determine the necessary and sufficient conditions for obtaining the various forms of imitative learning in animals.

\section{ACKNOWLEDGEMENTS}

Preparation of this article was supported by Grant MH 55118 from the National Institute of Mental Health and by Grant IBN 9414589 from the National Science Foundation.

\section{REFERENCES}

Akins, C. K., and T. R. Zentall. 1996. Imitative learning in male Japanese quail (Coturnix japonica) using the two-action method. Journal of Comparative Psychology 110:316-320.

Akins, C. K., and T. R. Zentall. 1998. Imitation in Japanese quail: The role of reinforcement of the demonstrator's response. Psychonomic Bulletin \& Review 5:694-697.

Akins, C. K., and T. R. Zentall. 1999. Imitative learning in Japanse quail depends on the motivational state of the observer at the time of observation, submitted. Armstrong, E. A. 1951. The nature and function of animal mimesis. Bulletin of Animal Behaviour 9:46-48.

Baer, D. M., R. F. Peterson, and J. A. Sherman. 1967. The development of imitation by reinforcing behavioral similarity to a model. Journal of the Experimental Analysis of Behavior 10:405-416. 
Ball, J. 1938. A case of apparent imitation in a monkey. Journal of Genetic Psychology 52:439-442.

Bandura, A. 1969. Social learning theory of identifictory processes. In D. A. Goslin, ed. Handbook of socialization theory and research, 213-262. Chicago: Rand-McNally.

Baptista, L. F., and L. Petrinovich. 1984. Social interaction, sensitive phases, and the song template hypothesis in the white-crowned sparrow. Animal Behaviour 32:172-181.

Bayroff, A. G., and K. E. Lard. 1941. Experimental social behaviour of animals. III. Imitational learning of white rats. Journal of Comparative and Physiological Psychology 37:165-171.

Boyd, R., and P. J. Richerson. 1988. An evolutionary model of social learning: The effect of spatial and temporal variation. In T. R. Zentall and B. G. Galef, Jr., eds., Social learning: Psychological and biological perspectives, 29-48. Hillsdale, NJ: Erlbaum.

Breuggeman, J. A. 1973. Parental care in a group of free-ranging rhesus monkeys. Folia Primatologica 20:178-210.

Bunch, G. B., and T. R. Zentall. 1980. Imitation of a passive avoidance response in the rat. Bulletin of the Psychonomic Society 15:73-75.

Bunyar, T., and L. Huber. 1999. Push or pull: An experimental study on imitation in marmosets. Animal Behaviour 54:817-831.

Byrne, R. W. 1994. The evolution of intelligence. In P. J. B. Slater and T. R. Halliday, eds, Behavior and evolution, 223-265. Cambridge: Cambridge University Press.

Byrne, R. W. 1995. The thinking ape. Oxford, UK: Oxford University Press.

Byrne, R. W., and A. E. Russon. 1998. Learning by imitation: A hierarchical approach. Behavioral and Brain Sciences 21:667-721.

Campbell, F. M., C. M. Heyes, and A. R. Goldsmith. 1999. Stimulus learning and response learning by observation in the European starling in a two-objective/two-action test. Animal Behaviour 58:151-158.

Chesler, P. 1969. Maternal influence in learning by observation in kittens. Science 166:901-903.

Church, R. M. 1957. Two procedures for the establishment of imitative behavior. Journal of Comparative and Physiological Psychology 50:315-318.

Clayton, D. A. 1978. Socially facilitated behavior. Quarterly Review of Biology 53:373-391.

Corson, J. A. 1967. Observational learning of a lever pressing response. Psychonomic Sciences 7:197-198.

Custance, D. M., and K. A. Bard. 1994. The comparative and developmental study of self-recognition and imitation: the importance of social factors. In S. T. Parkar, R. W. Mitchell and M. L. Boccia, eds. Self-awareness in 
humans and animals: developmental perspective, 207-226. Cambridge: Cambridge University Press.

Custance, D. M., A. Whiten, and K. A. Bard. 1995. Can young chimpanzees imitate arbitrary actions? Hayes and Hayes revised. Behaviour 132:839-858.

Custance, D. M., A. Whiten, and T. Fredman. 1998. Social learning of artificial fruit capuchin monkeys (Cebus apella). Journal of Comparative Psychology 113:13-23.

Davis, J. M. 1973. Imitation: A review and critique: In P. P. G. Bateson and P. H. Klopfer, eds. Perspectives in ethology, vol. 1, 43-72. New York: Plenum.

Davitz, J. R., and D. J. Mason. 1955. Socially facilitated reduction of a fear response in rats. Journal of Comparative and Physiological Psychology 48:149-151.

Dawson, B. V., and B. M. Foss. 1965. Observational learning in budgerigars. Animal Behaviour 13:470-474.

Del Russo, J. E. 1971. Observational learning in hooded rats. Psychonomic Science 24:37-45.

Del Russo, J. E. 1975. Observational learning of discriminative avoidance in hooded rats. Animal Learning and Behavior 3:76-80.

Denny, M. R., C. F. Clos, and R. C. Bell. 1988. Learning in the rat of a choice response by observation of S-S contingencies. In T. R. Zentall and B. G. Galef, Jr. eds., Psychological learning: Social and biological perspectives, 207-223. Hillsdale, NJ: Erlbaum.

Dorrance, B. R., and T. R. Zentall. 1999. Imitative learning in Japanese quail depends on the motivational state of the observer quail at the time of observation, submitted.

Dugatkin, L. A. 1992. Sexual selection and imitation: Females copy the male choice of others. American Nature 139:1384-1389.

Dugatkin, L. A. 1996. Copying and mate choice. In C. M. Heyes and B. G. Galef, eds., Social learning in animals: The roots of culture, 85-105. San Diego, CA: Academic Press.

Dugatkin, L. A., and J.-G. J. Godin. 1992. Reversal of female mate choice by copying. Proceedings of the Royal Society of London: Series B, 249:179-184.

Edwards, C. A., D. E. Hogan, and T. R. Zentall. 1980. Imitation of an appetitve discriminatory task by pigeons. Bird Behaviour 2:87-91.

Fiorito, G., and P. Scott. 1992. Observational learning in Octopus vulgaris. Science 256:545-546.

Fisher, J., and R. A. Hinde. 1949. Further observations on the opening of milk bottles by birds. British Birds 42:347-357.

Fragaszy, D. M., and E. Visalberghi, 1989. Social influences on the acquisition of tool using behavior in capuchin monkeys (Cebus apella). Journal of Comparative Psychology 103:159-170. 
Fragaszy, D. M., and E. Visalberghi. 1990. Social processes affecting the appearance of innovative behaviors in capachin monkeys. Folia Primatologica 54:155-165.

Fragaszy, D. M., and E. Visalberghi (1966). Social learning in monkeys: Primate "primacy" reconsidered. In C. M. Heyes and B. G. Galef, eds., Social Learning in animals: The roots of culture, 65-84. San Diego, CA: Academic Press.

Freud, S. 1933. New introductory lectures on psycho-analysis. New York: Norton.

Fritz, J., and K. Kotraschal. 1999. Social learning in common ravens (Corvus corax). Animal Behavior 54: 817-831.

Galef, B. G., Jr. 1988a. Communication of information concerning distant diets in a social, central-place foraging species: Ratus norvegicus. In T. R. Zentall and B. G. Galef, Jr., eds., Social learning: Psychological and biological perspectives, 119-139. Hillsdale, NJ: Erlbaum.

Galef, B. G., Jr. 1988b. Imitation in animals: History, definition, and interpretation of data from the psychological laboratory. In T. R. Zentall and B. G. Galef, Jr. eds., Social learning: Psychological and biological perspectives, 3-28. Hillsdale, NJ: Erlbaum.

Galef, B. G., Jr. 1996. Social enhancement of food preferences in Norway rats: A brief review. In C. M. Heyes and B. G. Galef, eds., Social learning in animals: The roots of culture, 49-64. San Diego, CA: Academic Press.

Galef, B. G., Jr., and P. J. Durlach 1993. Absence of blocking, overshadowing and latent inhibition in social enhancement of food preferences. Animal Learning \& Behavior 21:214-220.

Galef, B. G., Jr., L. A. Manzig, and R. M. Field. 1986. Imitation learning in budgerigars: Dawson and Foss (1965) revisited. Behavioral Processes 13:191-202.

Garcia, J., and R. A. Koelling. 1966. Relation of cue to consequence in avoidance learning. Psychonomic Science 4:123-124.

Gardner, E. L., and D. R. Engel. 1971. Imitational and social facilitatory respects of observational learning in the laboratory rat. Psychonomic Science 25:5-6.

Gardner, M., and C. M. Heyes. 1998, June. Limitation of the bidirectional control procedure. Paper preented at the Napoli Social Learning Conference, Naples, Italy.

Gewirtz, J. L. 1969. Mechanisms of social learning: Some roles of stimulation and behavior in early human development. In D. A. Goslin, ed., Handbook of socialization theory and research, 57-211. Chicago: Rand-McNally.

Gibson, J. 1979. The ecological approach to visual perception. Boston: Houghton Mifflin.

Groesbeck, R. W., and P. H. Duerfeld. 1971. Some relevant variables in observational learning of the rat. Psychonomic Science 22:41-43. 
Guillaume, P. 1971. Imitation in Children (E. P. Halperin, translator). Chicago: University of Chicago Press.

Harley, H. E., M. J., Xitco, Jr., H. L. Roitblat, and L. M. Herman. 1998, June. Imitation of human models by bottlenose dolphins. Paper presented at the Napoli Social Learning Conference, Naples, Italy.

Haruki, Y., and T. Tsuzuki. 1967. Learning of imitation and learning through imitation in the white rat. Annual of Animal Psychology 17:57-63.

Hayes, K. J., and C. Hayes. 1952. Imitation in a home-reared chimpanzee. Journal of Comparative and Physiological Psychology 45:450-459.

Henning, J. M., and T. R. Zentall. 1981. Imitation, social facilitation, and the effects of ACTH 4-10 on rats' barpress behavior. American Journal of Psychology 94:125-134.

Herbert, M. J., and C. M. Harsh. 1944. Observational learning by cats. Journal of Comparative Psychology 37:81-95.

Hess, E. H. 1973. Imprinting. New York: Van Nostrand Reinhold.

Heyes, C. M. 1994. Reflections on self-recognition in primates. Animal Behavior 47:909-919.

Heyes, C. M. 1996. Genuine imitation: In C. M. Heyes and B. G. Galef, eds., Social learning in animals: The roots of culture, 371-389. San Diego, CA: Academic Press.

Heyes, C. M., and G. R. Dawson. 1990. A demonstration of observational learning in rats using a bidirectional control. Quarterly Journal of Experimental Psychology 42B:59-71.

Heyes, C. M., E. Jaldow, and G. R. Dawson. 1994. Imitation in rats: Conditions of occurrence in a bidirectional control procedure. Learning and Motivation 25:276-298.

Hinde, R. A. ed. 1969. Bird vocalizations. Cambridge, UK: Cambridge University Press.

Hogan, D. E. 1988. Learned imitation by pigeons. In T. R. Zentall and B. G. Galef, Jr. eds., Social learning: Psychological and biological perspectives, 225-238. Hillsdale, NJ: Erlbaum.

Hoogland, J. L., and P. W. Sherman. 1976. Advantages and disadvantages of bank swallow (Riparia riparia) coloniality. Ecological Monographs 46:33-58.

Howard, M. L., and M. Keenan. 1993. Outline for a functional analysis of imitation in animals. Psychological Record 43:185-204.

Huber, L. 1998. Movement imitation as faithful copying in the absence of insight. Behavioral and Brain Sciences 21:694.

Huffman, M. A. 1996. The study of nonhuman primate culture in Japan. In C. M.

Heyes and B. G. Galef, eds., Social learning in animals: The roots of culture, 267-289. San Diego, CA: Academic Press.

Hull, C. L. 1943. Principles of behavior. New York: Appleton. 
Jacoby, K. E., and M. E. Dawson. 1969. Observation and shaping learning: A comparison using Long-Evans rats. Psychonomic Science 16:257-258.

John, E. R., P. Chesler, F. Bartlett, and I. Victor. 1968. Observational learning in cats. Science 159:1489-1491.

Jones, S. S. 1996. Imitation or exploration: Young infants' matching of adults' oral gestures. Child Development 67:1952-1969.

Kaiser, D. H., T. R. Zentall, and B. G. Galef, Jr. 1997. Can imitation in pigeons be explained by local enhancement together with trial-and-error learning? Psychological Science 8:459-465.

Kohn, B. 1976. Obsiervation and discrimination learning in the rat: Effects of stimulus substitution. Learning and Motivation 7:303-312.

Kohn, B., and M. Dennis. 1972. Observation and discrimination learning in the rat: Specific and nonspecific effects. Journal of Comparative and Physiological Psychology 78:292-296.

Kovach, J. K., and E. H. Hess. 1963. Imprinting: Effects of painful stimulation upon the following response. Journal of Comparative and Physiological Psychology 56:461-464.

Lefebvre, L., and B. Palameta. 1988. Mechanisms, ecology, and population diffusion of socially learned food-funding behavior in feral pigeons. In $\mathrm{T}$. R. Zentall and B. G. Galef, Jr. eds., Social learning: Psychological and biological perspectives, 141-164. Hillsdale, NJ: Erlbaum.

Levine, J. M., and T. R. Zentall. 1974. Effect of conspecific's presence on deprived rats performance: Social facilitation vs. distraction/imitation. Animal Learning and Behavior 2:119-122.

Lore, R., A. Blanc, and P. Suedfeld. 1971. Empathic learning of a passive avoidance response in domesticated Rattus norvegicus. Animal Behaviour 19:112-114.

Lorenz, K. 1935. Der kumpanin der umvelt des vogels: die artgenosse als ausloesendesmoment socialer verhaltenweisen. Journal fur Ornithologie 83:137-213, 289-413.

Marler, P. 1970. A comparative approach to vocal learning. song development in white-crowned sparrows. Journal of Comparative and Physiological Psychology 71:1-25.

Meltzoff, A. N. 1996. The human infant as imitative generalist: A 20-year progress report on infant imitation with impliations for comparative psychology. In C. M. Heyes and B. G. Galef, eds., Social learning in animals: The roots of culture, 347-370. San Diego, CA: Academic Press.

Meltzoff, A. N., and M. K. Moore. 1989. Imitation in newborn infants: Exploring the range of gestures imitated and the underlying mechanisms. Developmental Psychology 25:954-962.

Miles, H. L., R. W. Mitchell, and S. E. Harper. 1996. Simon says: The development of imitation in an encultured orangutan. In A. E. Russon, K. E. Bard, 
and S. T. Parker, eds., Reaching into thought: The minds of the great apes, 278-299. Cambridge, UK: Cambridge University Press.

Miller, N. E., and J. Dollard. 1941. Social learning and imitation. New Haven, CT: Yale University Press.

Mineka, S., and M. Cook. 1988. Social learning and the acquisition of snake fear in monkeys. In T. R. Zentall and B. G. Galef, Jr. eds., Social learning: Psychological and biological perspectives, 51-75. Hillsdale, NJ: Erlbaum.

Mitchell, C. J., C. M. Heyes, and G. R. Dawson. 1999. Limitations of a bidirectional control procedure for the investigation of imitation in rats: Odour cues on the manipulandum. Quarterly Journal of Experimental Psychology 52:193-202.

Mitchell, R. W. 1987. A comparative-developmental approach to understanding imitation. In P. P. G. Bateson and P. H. Klopfer, eds., Perspectives in ethology, Vol. 7, 183-215. New York: Plenum.

Mitchell, R. W. 1993. Mental models of mirror-self-recognition: Two theories. New Ideas in Psychology 11:295-325.

Moore, B. R. 1992. Avian movement imitation and a new form of mimicry: Tracing the evolution of a complex form of learning. Behaviour 122: 231-263.

Moore, B. R. 1996. The evolution of imitative learning. In C. M. Heyes and G. G. Galef, eds., Social learning in animals: The roots of culture, 245-265. San Diego, CA: Academic Press.

Morgan, C. L. 1900. Animal behavior. London: Arnold.

Morrison, B. J., and W. F. Hill. 1967. Socially facilitated reduction of the fear response in rats raised in groups or in isolation. Journal of Comparative and Physiological Psychology 63:71-76.

Nottebohm, F. 1970. Ontogeny of bird song. Science 167:950-956.

Oldfield-Box, H. 1970. Comments on two preliminary studies of "observation" learning in the rat. Journal of Genetic Psychology 116:45-51.

Palameta, B., and L. Lefebvre. 1985. The social transmission of a food-finding technique in pigeons: What is learned? Animal Behavior 33:892-896.

Pepperberg, I. M. 1986. Acquisition of anomalous communicatory systems: Implications for studies on interspecies communication. In R. Schusterman, J. Thomas, and F. Wood, eds., Dolphin behavior and cognition: Comparative and ethological aspects, 289-302. Hillsdale, NJ: Erlbaum.

Pepperberg, I. M. 1988. The importance of social interaction and observation in the acquisition of communicative competence: Possible parallels between avian and human learning. In T. R. Zentall and B. G. Galef, Jr. eds., Social learning: Psychological and biological perspectives, 279-299. Hillsdale, NJ: Erlbaum.

Pepperberg, I. M. 1990. Referential mapping. Applied Psycholinguistics $11: 23-44$.

Piaget, J. 1955. The child's construction of reality. London: Routledge. 
Powell, R. W. 1968. Observational learning vs shaping: A replication. Psychonomic Science 10:263-364.

Powell, R. W., and R. Burns. 1970. Visual factors in observational learning with rats. Psychonomic Science 21:47-48.

Powell, R. W., D. Saunders, and W. Thompson. 1969. Shaping, autoshaping, and observational learning with rats. Psychonomic Science 13:167-168.

Ray, E. D., and C. M. Heyes. 1998, June. Implications of a contemporary learning theory for social learning research. Paper presented at Napoli Social Learning conference, Naples, Italy.

Reissland, N. 1988. Neonatal imitation in the first hour of life: Observations in rural Nepal. Development Psychology 24:464-469.

Roberts, D. 1941. Imitation and suggestion in animals. Bulletin of Animal Behaviour 1:11-19.

Russon, A. E., and B. M. F. Galdikas. 1993. Imitation in ex-captive orangutans (Pongo pymaeus). Journal of Comparative Psychology 107:147-161.

Russon, A. E., and B. M. F. Galdikas. 1995. Constraints on great apes' imitation: Model and action selectivity in rehabilitant orangutan (Pongo pygmaeus) imitation. Journal of Comparative Psychology 109:5-17.

Sanavio, E., and U. Savardi. 1979. Observational learning in Japanese quail. Behavioral Proceses 5:355-361.

Sanavio, E., and U. Savardi. 1980. Observational learning of a discriminative shuttlebox avoidance by rats. Psychological Reports 44:1151-1154.

Sordahl, T. A. 1981. Slight of wing. Natural History 90:42-49.

Spence, K. W. 1937. The differential response in animals to stimuli varying within a single dimension. Psychological Review 44:430-444.

Stimbert, V. E. 1970. A comparison of learning based on social and nonsocial discriminative stimuli. Psychonomic Science 20:185-186.

Strupp, B. J., and D. A. Levitsky. 1984. Social transmission of food preferences in adult hooded rats (Rattus norvegicus). Journal of Comparative Psychology 98:257-266.

Tanner, J., and R. W. Byrne. 1999. Imitation in a captive lowland gorilla: A spontaneous experiment. Unpublished manuscript, University of St. Andrewsm Fife, Scotland..

Thorpe, W. H. 1961. Bird song: The biology of vocal communication and expression in birds. Cambridge, MA: Harvard University Press.

Thorpe, W. H. 1963. Learning and instinct in animals, 2nd ed. Cambridge, MA: Harvard University Press.

Thorpe, W. H. 1967. Vocal imitation and antiphonal song and its implications. In D. W. Snow, ed., Proceedings of the XVI International Ornithological Congress, 245-263. Oxford, UK: Blackwell.

Tinbergen, N. 1960. The herring gull's world. Garden City, NY: Doubleday. 
Todt, D. 1975. Social learning of vocal patterns and modes of their applications in Grey parrots. Zeitschrift für Thierpsychologie 39:178-188.

Tolman, C. W. 1964. Social facilitation of feeding behaviour in the domestic chick. Animal Behaviour 12:245-251.

Tomasello, M. 1990. Cultural transmission in the tool use and communicatory signaling of chimpanzees? In S. Parker and K. Gibson, eds., "Language", and intelligence in monkeys and apes: Comparative developmental perspectives, 271-311. Cambridge, UK: Cambridge University Press.

Tomasello, M. 1996. Do apes ape? In C. M. Heyes and B. G. Galef, eds., Social learning in animals: The roots of culture, 319-346. San Diego, CA: Academic Press.

Tomasello, M., and J. Call. 1994. The social cognition of monkeys and apes. Yearbook of Physical Anthropology 37:273-305.

Tomasello, M., D. Gust, and T. Frost. 1989. A longitudinal investigation of gestural communication in young chimpanzees. Primates 30:35-50.

Tomasello, M., A. C. Kruger, and H. H. Ratner. 1993. Cultural learning. Behavioral and Brain Sciences 16:495-552.

Tomasello, M., S. Savage-Rumbaugh, and A. C. Kruger. 1993. Imitative learning of actions on objects by children, chimpanzees, and enculturated chimpanzees. Child Development 64:1688-1705.

Turner, J. R. G. 1984. Mimicry: The palatability spectrum and its consequences. In R. I. Vane-Wright and P. R. Ackery, eds. The biology of butterflies, 141-161. New York: Academic Press.

Tyrrell, D. J., M. C. Zingaro, and K. L. Minard. 1993. Learning and transfer of identity-difference relationships by infants. Infant Behavior and Devlopment 16:43-52.

Vanayan, M., H. Robertson, and G. B. Biederman. 1985. Observational learning in pigeons: The effects of model proficiency on observer performance. Journal of General Psychology 112:349-357.

Visalberghi, E. 1093. Tool use in a South American monkey species: An overview of characteristics and limits of tool use in Cebus apella. In A. Bethelet and J. Chavaillon, eds, The use of tools by human and nonhuman primates. Cambridge, UK: Oxford University Press.

Warden, C. J. and T. A. Jackson. 1935. Imitative behavior in the rheus monkey. Journal of Genetic Psychology 46:103-125.

Weigle, P. D., and E. V. Hanson. 1980. Observation learning and the feeding behavior of the red squirrel (Tamiasciurus hudsonicus): The ontogeny of optimization. Ecology 61:213-218.

Whiten, A. 1998. Transmission mechanisms in primate cultural evolution. Trends in Ecology and Evolution 4:61-62. 
Whiten, A. (in press). Chimpanzee cognition and the question of mental re-representation. In D. Sperber, ed., Metarepresentation. Oxford, UK: Oxford University Press.

Whiten, A., and D. Custance. 1996. Studies of imitation in chimpanzees and children. In C. M. Keyes and B. G. Galef, eds., Social learning in animals: The roots of culture, 291-318. San Diego, CA: Academic Press.

Whiten, A., D. M. Custance, J.-C. Gomez, P. Teixidor, and K. A. Bard. 1996. Imitative learning of artificial fruit processing in children (Homo sapiens) and chimpanzees (Pan troglodytes). Journal of Comparative Psychology 110:3-14.

Whiten, A., and R. Ham. 1992. On the nature and evolution of imitation in the animal kingdom: Reappraisal of a century of research. In P. J. B. Slater, J. S. Rosenblatt, C. Beer, and M. Milinski, eds. Advances in the study of behaviour, Vol. 21, 239-283. New York: Academic Press.

Will, B., B. Pallaud, M. Soczka, and S. Manikowski. 1974. Imitation of lever pressing "strategies" during the operant conditioning of albino rats. Animal Behaviour 22:664-671.

Xitco, M. J., Jr., H. E. Harley, and R. L. Brill. 1998, June. Action level imitation by bottlenose dolphins. Paper presented at the Napoli Social Learning Conference, Naples, Italy.

Zajonc, R. B. 1965. Social facilitation. Science 149:269-274.

Zentall, T. R. 1996. An analysis of imitative learning in animals. In C. M. Heyes and B. G. Galef, eds. Social Learning in Animals: The Roots of Culture, 221-243. San Diego, CA: Academic Press.

Zentall, T. R., C. A. Edwards, and D. E. Hogan. 1983. Pigeons' use of identity. In M. L. Commons, R. J. Herrnstein and A. Wagner, eds., The quantitative analyses of behavior: Vol. 4. Discrimination processes, 273-293. Cambridge, MA: Ballinger.

Zentall, T. R., C. A. Edwards, B. S. Moore, and D. E. Hogan. 1981. Identity: The basis for both matching and oddity learning in pigeons. Journal of Experimental Psychology: Animal Behavior Processes 7:70-86.

Zentall, T. R., and D. E. Hogan. 1975. Key pecking in pigeons produced by pairing key light with inaccessible grain. Journal of the Experimental Analysis of Behavior 23:199-206.

Zentall, T. R., and D. E. Hogan. 1976. Imitation and social facilitation in the pigeon. Animal Learning \& Behavior 4:427-430.

Zentall, T. R., and J. M. Levine. 1972. Observational learning and social facilitation in the rat. Science 178:1220-1221.

Zentall, T. R., J. Sutton, and L. M. Sherburne. 1996. True imitative learning in pigeons. Psychological Science 7:343-346. 